\section{THU0283 RESILIENCE AND ASSOCIATED FACTORS AMONG WOMEN WITH SYSTEMIC LUPUS ERYTHEMATOSUS}

P. Munguía-Realpozo $^{1}$, M. García-Carrasco ${ }^{2,3}$, J. León-Vázquez $^{4}$, M.F. Balcazar-Vargas ${ }^{2}$, E.A. Jiménez-Herrera ${ }^{4}$, I. Etchegaray-Morales ${ }^{4}$, C. Mendoza Pinto 2,4 . ${ }^{1}$ Rheumatology, Hospital de Especialidades Centro Médico Nacional "La Raza" IMSS, Cd. México; ${ }^{2}$ Immunology and Rheumatology, Medicine School, BUAP; ${ }^{3}$ Systemic Autoimmune Diseases Research Unit, Hgr 36-Cibior, IMSS; ${ }^{4}$ Systemic Autoimmune Diseases Research Unit, Hgr-36-Cibior Imss, Puebla, Mexico

Background: Resilience is the human capacity to respond positively to adverse situations, enabling individuals to achieve, maintain, or recover physical or emotional health after devastating illness, losses, or other stressful situations. Resilience may be an important factor in health promotion.

Objectives: The aim of this study was to evaluate resilience and possible associated factors in patients with systemic lupus erythematosus (SLE).

Methods: In a cross-sectional study, 123 SLE women and 133 age-matched controls were evaluated using the Spanish version of Wagnild and Young's Resilience Scale (WYRS, 1993). SLE patients underwent a structured interview to collect sociodemographic data, including socioeconomic status measured by the Graffar method. The Center for Epidemiologic Studies Depression Scale (CES-D) was used to measure depressive symptoms.

Results: The median age and duration disease of SLE women was 45 (IQR: 34-54) years and 11 (IQR: 8-15) years, respectively. Thirty-three percent had depressive symptoms (CESD score $\geq 16$ ). Resilience scores did not differ between patients and controls (median 80 IQR: $75-87$ vs. 80 IQR: $74-85$; $p=0.38$ ). However, patients with SLE had a higher personal competence factor than controls. Personal competence factor correlated negatively with age and socioeconomic status $(R=$ -0.220 , $p: 0.05$ and $R=-0.357, p<0.001)$ and positively with educational level $(R=0.324, p<0.001)$. Less resilience (lower WYRS scores) correlated with depressed mood (higher CESD scores) $(R=-0.537, p<0.001)$.

Conclusions: Resilience in patients with SLE did not differ from that of controls. Age, socioeconomic status and depressive symptoms correlated with low resilience. Educational level correlated with higher resilience.

References:

[1] Windle G, Bennett K, Noyes J. A methodological review of resilience measurement scales. Health Qual Life Outcomes. 2011; 9(8): 1-18.

[2] Pousa DA, Revoredo LS, Vilar MJ and Chavez EM. Resilience and Treatment Adhesion in Patients with Systemic Lupus Erythematosus. Open Rheumatol J. $2014 ; 8$ : 1-8.

Acknowledgements: We would like to thank David Buss for his valuable guidance and advice during this project.

Disclosure of Interest: None declared

DOI: 10.1136/annrheumdis-2017-eular.2481

\section{THU0284 PREDICTING SURVIVAL IN 6240 PATIENTS WITH PRIMARY SJÖGREN' SYNDROME (BIG DATA SJÖGREN PROJECT)}

P. Brito-Zerón ${ }^{1,2}$, N. Acar-Denizli ${ }^{3}$, M. Zeher ${ }^{4}$, A. Rasmussen ${ }^{5}, \mathrm{X} . \mathrm{Li}^{6}$, C. Baldini ${ }^{7}$, J.-E. Gottenberg ${ }^{8}$, D. Danda ${ }^{9}$, L. Quartuccio ${ }^{10}$,

G. Hernandez-Molina ${ }^{11}$, A.A. Kruize ${ }^{12}$, S.-H. Park ${ }^{13}$, M. Kvarnström ${ }^{14}$

S. Praprotnik ${ }^{15}$, D. Sene ${ }^{16}$, E. Bartoloni ${ }^{17}$, R. Solans ${ }^{18}$, T. Mandl ${ }^{19}$, Y. Suzuki ${ }^{20}$,

M. Rischmueller ${ }^{21}$, G. Nordmark ${ }^{22}$, G. Fraile ${ }^{23}$, A. Sebastian ${ }^{24}$, H. Bootsma ${ }^{25}$,

T. Nakamura ${ }^{26}$, V. Valim ${ }^{27}$, R. Giacomelli ${ }^{28}$, R. Seror ${ }^{29}$

V. Devauchelle-Pensec ${ }^{30}$, B. Hofauer $^{31}$, M. Bombardieri ${ }^{32}$, V. Trevisani ${ }^{33}$, D. Hammenfors ${ }^{34}$, R. Priori ${ }^{35}$, S.G. Pasoto ${ }^{36}$, J. Morel ${ }^{37}$, S. Retamozo ${ }^{38}$, T.A. Gheita ${ }^{39}$, F. Atzeni ${ }^{40}$, C. Vollenveider ${ }^{41}$, X. Mariette ${ }^{29}$, M. Ramos-Casals ${ }^{2}$ on behalf of the EULAR-SS Task Force Big Data Consortium. ${ }^{1} \mathrm{Hosp}$ CIMA-Sanitas; ${ }^{2}$ Hosp Clínic, Barcelona, Spain; ${ }^{3}$ Msgsü, Istanbul, Turkey; ${ }^{4}$ Univ, Debrecen, Hungary; ${ }^{5}$ OMRF, Oklahoma, United States; ${ }^{6}$ Anhui Hosp, Hefei, China; ${ }^{7}$ Univ, Pisa, Italy; ${ }^{8}$ Univ, Strasbourg, France; ${ }^{9} \mathrm{CMC}$, Vellore, India; ${ }^{10}$ Santa Maria, Udine, Italy; ${ }^{11}$ INCMNSZ, México, Mexico; ${ }^{12}$ UMC, Utrecht, Netherlands; ${ }^{13}$ Catholic Univ, Seoul, Korea, Republic Of; ${ }^{14}$ Karolinska Instit, Stockholm, Sweden; ${ }^{15}$ UMCL, Ljubljana, Slovenia; ${ }^{16}$ Lariboisière Hosp, Paris, France: ${ }^{17}$ Univ, Perugia, Italy; ${ }^{18}$ Hosp Vall Hebron, Barcelona, Spain; ${ }^{19}$ Lund Univ, Malmö, Sweden; ${ }^{20}$ Univ, Kanazawa, Japan; ${ }^{21}$ TQEH, Adelaide, Australia; ${ }^{22}$ Univ, Uppsala, Sweden; ${ }^{23}$ Hosp Ramón Cajal, Madrid, Spain; ${ }^{24}$ Med Hosp, Wroclaw, Poland; ${ }^{25}$ Univ, Groningen, Netherlands: ${ }^{26}$ Univ, Nagasaki, Japan: ${ }^{27}$ UFES, Vitória, Brazil; ${ }^{28}$ Univ, L'Aquila, Italy; ${ }^{29}$ Univ Paris Sud, Paris; ${ }^{30}$ Univ, Brest, France; ${ }^{31}$ TUM, München, Germany; ${ }^{32}$ QMUL, London, United Kingdom; ${ }^{33}$ UNIFESP, Sao Paulo, Brazil; ${ }^{34}$ Haukeland Hosp, Bergen, Norway; ${ }^{35}$ Sapienza Univ, Rome, Italy; ${ }^{36}$ USP, Sao Paulo, Brazil; ${ }^{37}$ Univ, Montpellier, France;

${ }^{38}$ INICSA, Cordoba, Argentina; ${ }^{39}$ Univ, Cairo, Egypt; ${ }^{40}$ L.Sacco Univ Hosp, Milan, Italy; ${ }^{41}$ German Hosp, Buenos Aires, Argentina

Objectives: To correlate the main features at diagnosis with survival in a multicenter international cohort of patients with primary Sjögren syndrome (SS). Methods: The Big Data Sjögren Project was formed in 2014 to take a "highdefinition" picture of the main features of primary SS by merging international SS databases (9302 consecutive patients recruited from 21 countries of the 5 continents)

Results: Data about survival at the last visit was available in 6240 patients (5831 women, mean age at diagnosis of $53 y$ rs, $82 \%$ White). After a mean follow-up of 7.1yrs, $407(6 \%)$ patients died. Patients who died were more frequently men (10\% vs $6 \%, p=0.004)$ and White $(96 \%$ vs $81 \%, p<0.001)$, had a higher mean age at diagnosis (64 vs 52yrs. $p<0.001$ ) and a higher frequency of abnormal ocular $(95 \%$ vs $87 \%, p<0.001)$ and oral $(90 \%$ vs $79 \%, p<0.001)$ tests in comparison with survivors; sicca symptoms and salivary gland biopsy were unrelated to survival. Immunologically, patients who died showed a higher frequency of RF (52\% vs $46 \%, p=0.039)$ and cryoglobulins $(18 \%$ vs $7 \%, p<0.001)$. Logistic regression identified as independent variables the age at diagnosis (OR 1.07), male gender (OR 1.77), abnormal ocular (OR 5.79) and oral (OR 2.10) tests, and cryoglobulins (OR 3.10). Time-adjusted multivariate Cox proportional-hazards regression analysis confirmed age at diagnosis (OR 1.12, Cl95\% 1.10-1.14), male gender (OR 2.12, Cl95\% 1.36-3.29) and cryoglobulins (OR 2.43, Cl95\% 1.72-3.42) as independent variables related to death.

Conclusions: An older age at diagnosis, male gender and serum cryoglobulins were the principal risk factors associated with death in this large multiethnic cohort of patients with primary SS.

Disclosure of Interest: None declared

DOI: 10.1136/annrheumdis-2017-eular.4446

\section{THU0285 USE OF CONTRACEPTIVE METHODS IN A SINGLE CENTER COHORT OF SYSTEMIC LUPUS ERYTHEMATOSUS FROM ARGENTINA}

R.A. Gomez, F.M. Paniego, M. Garcia Carrasco, D. Dubinsky, G. Nasswetter. Rheumatology, Hospital de Clinicas Jose de San Martin, Ciudad Autónoma de Buenos Aires, Argentina

Background: Systemic Lupus Erythematosus (SLE) may associate with flares, an unfavorable course and the need of teratogenic treatment during pregnancy. Not every contraceptive method (CM) may be used in this patients. There are guidelines for their use according to existing morbidity and SLE activity ${ }^{1}$. Objectives: Describe the use of CM in a cohort of outpatients with SLE

Methods: Descriptive, observational, cross-sectional study. Patients $\geq 16$ years old with SLE (SLICC 2012) and $\geq 1$ visit during the last year of our database were included. Those with menopause were excluded. We analyzed demographic data; disease duration; antiphospholipid antibodies ( $\mathrm{aPL}$ ) and antiphospholipid syndrome (APS) (Sapporo 2006); socioeconomic status (Graffar scale); disease activity (SELENA-SLEDAI) and accrual damage (SLICC); use of teratogens: Methotrexate (MTX), Mycophenolate (MYC), Cyclophosphamide (CYC), biologic drugs (BD), self-reported sexual status (active/non active) and $\mathrm{CM}$ : intrauterine device (IUD), condom (Cdm) and hormonal contraceptive pill (CP).

Results: $132 / 219$ were included. Female $91.6 \%$, age: $30.1(16-49)$ years, disease duration: 38.5 (1-324) months, SELENA-SLEDAI: 3.5 (0-29), SLICC: 0.5 (0-5).

Sexual status and use of CM: were available for 120 patients. Sexually active (SA) $73 \%$, female $77 \%$. CM in SA patients: $74 \%$ : $5 \%$ CP, $77 \%$ Cdm and $18 \%$ IUD. $C M$ in female patients stratified by Graffar (GF): GF I $(n=1)$ : none SA; GF II: $(n=19): 84 \%$ SA, $75 \%$ of them used CM $(75 \%$ Cdm, $25 \%$ IUD); GF III $(n=33)$ : $82 \%$ sexually active, $81 \%$ used CM ( $86 \% \mathrm{Cdm}, 14 \%$ IUD), GF IV ( $n=41) 75 \%$ SA, $68 \%$ used CM (9\% CP, $71 \% \mathrm{Cdm}, 19 \%$ IUD), GF V $(n=4) 100 \%$ SA, $50 \%$ used CM (50\% CP, 50\% Cdm); GF NA ( $\mathrm{n}=11) 55 \%$ SA, $83 \%$ used CM (80\% Cdm, $20 \%$ IUD).

CM in female patients stratified by SLE activity: SLEDAI <3 ( $n=62): 76 \%$ SA, $77 \%$ of them used CM (3\% CP, 83\% Cdm, 14\% IUD); SLEDAI 3-12 ( $n=42): 81 \%$ SA, $76 \%$ used CM (8\% CP, 69\% Cdm, 23\% IUD); SLEDAI >12 ( $n=60): 60 \%$ SA, $67 \%$ used CM $(100 \% \mathrm{Cdm})$.

CM in female patients using teratogenic drugs: MTX $(n=8): 100 \%$ SA, $75 \%$ of them used CM (17\% CP, 66\% Cdm, 17\% IUD); MYC ( $n=20): 80 \%$ SA, $88 \%$ used CM (71\% Cdm, $29 \%$ IUD); CYC ( $n=3): 33 \%$ SA, $100 \%$ used CM (100\% Cdm); Belimumab ( $n=1): 100 \%$ SA, $100 \%$ surgical CM (tubectomy)

aPL was evaluated in 96/132 patients. 17/96 had positive aPL and 5/17 fulfilled APS criteria. 2/17 patients had no CM data available, none with APS. 9/17 patients with aPL were female, $55 \%$ SA and all of them used $\mathrm{Cdm}$. $3 / 5$ patients with APS were female, all SA, $66 \%$ used Cdm.

Conclusions: Condom was the most reported $\mathrm{CM}$. Use of $\mathrm{CM}$ was more frequent in the upper social stratus (GF I-III) respect to the lower (GF IV-V).

The proportion of sexually active female patients was similar in those using teratogenic drugs compared with who had not used them.

The self-reported sexual status was similar despite of SLEDAI stratification, being similar in patients with low or high disease activity.

We deem necessary education, counseling and evaluation of use of the CM in every visit of patients with SLE.

References:

[1] Andreoli L, Bertsias GK, Agmon-Levin N, et al. Ann Rheum Dis, doi:10.1136/annrheumdis-2016-209770.

Disclosure of Interest: None declared

DOI: 10.1136/annrheumdis-2017-eular.3922 